\title{
A Pilot Study Evaluating the Effectiveness and Safety of Daikenchuto (TJ-100) for the Treatment of Postoperative Abdominal Pain or Bloating in Patients Undergoing Hepatectomy: Study Protocol for a Randomized, Open, Controlled Trial
}

\author{
SUSUMU EGUCHI, MASAAKI HIDAKA, AKIHIKO SOYAMA, TAKANOBU HARA, TOTA KUGIYAMA, \\ TAKASHI HAMADA, TAKAYUKI TANAKA, HAJIME MATSUSHIMA, TOMOHIKO ADACHI, \\ YUSUKE INOUE, SHINICHIRO ITO AND KENGO KANETAKA
}

Department of Surgery, Nagasaki University Graduate School of Biomedical Sciences, Nagasaki 852-8501, Japan

Received 7 July 2019, accepted 26 September 2019

J-STAGE advance publication 6 August 2021

Edited by YOSHITO AKAGI

\begin{abstract}
Summary: This study is being performed to evaluate the effectiveness and safety of TJ-100 TSUMURA Daikenchuto (DKT) Extract Granules in preventing post-hepatectomy digestive symptoms, and to examine the effects of DKT on small intestinal mucosal atrophy using diamine oxidase (DAO) and glucagon-like peptide-2 (GLP-2) activities. This is a randomized, open, controlled trial using patients treated with usual care as the control group. Patients who meet the inclusion criteria are randomized to the study groups. Eligible patients are randomized to the DKT therapy group (DKT administration for 14 days postoperatively or until the day of discharge if a patient leaves the hospital less than 14 days after the surgery) or the usual care group (no administration of DKT (ratio 1:1). Using the NRS (numeric rating scale) as an indicator, we will attempt to show whether DKT is effective for abdominal pain and bloating after surgery by comparing both groups. We will also attempt to evaluate postoperative small intestinal mucosal atrophy using DAO and GLP-2 activities in the serum, and postoperative nutrient absorption using nutrient assessment indicators. This study is being conducted according to the CONSORT statement. A consent form was signed by all participants, and the study protocol has been approved by the Central Review Board and Local Ethics Committee (CRB7180001).
\end{abstract}

Keywords Daikenchuto, DKT, numeric rating scale, diamine oxidase, glucagon-like peptide-2

\section{INTRODUCTION}

Daikenchuto [DKT] is a traditional Japanese medicine. TJ-100 TSUMURA Daikenchuto Extract Granules consist of a dried extract of a mixture containing processed ginger, ginseng, zanthoxylum fruit, and maltose. It is clinically used to treat postoperative symptoms accompanied by abdominal pain and bloating sensation such as paralytic ileus and bowel move- ment disturbance, and the reported action mechanisms are enhanced gastrointestinal motility [1-3], increased intestinal blood flow $[4,5]$ and anti-inflammatory effects $[6,7]$. Previous studies reported postoperative effects of DKT as shortening of the time to first defecation [8], first gas passage [9], and oral intake recovery [10] after abdominal surgery.

DKT has also been reported to accelerate first bowel movements and reduce inflammation in select-

Corresponding Author: Susumu Eguchi, M.D., Ph.D., Department of Surgery, Nagasaki University Graduate School of Biomedical Sciences, 1-7-1 Sakamoto, Nagasaki, Nagasaki 852-8501, Japan. Tel: +81-95-819-7316, Fax: +81-95-819-7319, E-mail: sueguchi@nagasaki-u.ac.jp

Abbreviations: Alb, Albumin; Al-P, Alkaline phosphatase; ALT, Alanin-aminotransferase; AST, Aspartate transaminase; CRC, clinical research coordinators; CRF, case report form; DAO, Diamine oxidase; DKT, Daikenchuto; GGTP, Gamma-glutamyl transpeptidase; GLP-2, Glucagon-like peptide-2; ICG, Indocyanine green; NRS, Numeric Rating Scale; POD, postoperative day; PT, Prothrombin. time; RBP, Retinol-binding protein; SPIRIT, Standard Protocol Items: Recommendations for Interventional Trials; T-Bil, Total bilirubin; Tf, Transferrin; TTR, Transthyretin; 
ed patients undergoing hepatectomy $[11,12]$. However, abdominal pain and bloating following hepatectomy has not been investigated. In addition, its mechanism of action has not yet been defined in humans due to the difficulty in setting objective parameters for assessment of abdominal pain and bloating. A recent study found that oral and enteric caloric intake after liver transplantation was facilitated by DKT in a randomized controlled trial [13]. In addition to these effects, the intestinal mucosal protective effect that has been reported by basic research leads us to expect that DKT will be effective in preventing postoperative intestinal mucosal atrophy, impairment of intestinal immunity, and also impairment of nutrient absorption capacity [14-17]. However, these effects have not yet been proven by clinical research.

Diamine oxidase (DAO) and glucagon-like peptide-2(GLP-2)activities are used as evaluation indicators reflecting the state of the small intestinal mucosa [18-20] and were used also in our department to evaluate the effects of early enteral alimentation after liver transplantation on the villi in the small intestine. Using DAO and GLP-2 activities in the serum, we are conducting this exploratory study to evaluate the effectiveness and safety of DKT in preventing post-hepatectomy digestive symptoms and to examine the effects of DKT on small intestinal mucosal atrophy.

We are evaluating the effectiveness and safety of DKT in preventing post-hepatectomy digestive symptoms using the NRS (numeric rating scale) and are performing an exploratory study of the effects of DKT on small intestinal mucosal atrophy by examining DAO and GLP-2 activities. The results of the study may provide clinical information on the effectiveness and safety of DKT and allow elucidation of the mechanism of action of DKT in clinical practice. In addition, our results are expected to facilitate development of improved treatments for the damage to the intestinal mucosa and accompanying impairment of nutrient absorption capacity after gastroenterological surgery.

\section{METHODS}

\section{Inclusion criteria}

The criteria for provisional registration are as follows: Selection criteria at the time of obtaining informed consent

1) Patient who are scheduled to undergo hepatectomy ( $>=$ partial hepatectomy) due to liver cancers

2) Liver Damage A (Liver Damage is a score advocated by the Liver Cancer Study Group of
Japan as an alternative to the Child-Pugh score, which is defined by preoperative measurements of ascites, serum bilirubin level, serum albumin level, indocyanine green retention rate at 15 minutes, and prothrombin activity) [21]

3) Age: $>=20$ years

4) Patient who are able to orally take Daikenchuto (DKT)

5) Adequate organ functions and operable patient

6) Patient able to provide written informed consent

Selection criteria at the time of enrollment

1) Abdominal pain or bloating on postoperative day 1

2) Patients who have undergone hepatectomy $(>=$ partial hepatectomy)

\section{Exclusion criteria}

1) Emergency surgery

2) History of hepatectomy

3) Patients scheduled to undergo partial resection of the liver due to metastatic liver cancer

4) Patients who have constitutional ICG (indocyanine green) excretory defect

5) History of gastrointestinal surgery (excluding appendectomy)

6) Patients who are scheduled to undergo gut surgery with hepatectomy (including biliary reconstruction)

7) Patients with inflammatory bowel diseases such as ulcerative colitis or Crohn disease

8) History of stoma

9) Patients who are scheduled for chemotherapy, radiation therapy or radiofrequency ablation therapy within 4 weeks before enrollment, during surgery or within 14 days after surgery (during individual study period)

10) Patients who took kampo medicine or lactobacillus preparation within 4 weeks before enrollment

11) Patients who are pregnant, possibly pregnant, considering pregnancy or lactating

12) Patient who are determined not applicable for the study by the physician

13) Prohibited drugs: drugs that are known to promote bowel movement are prohibited during the protocol treatment such as erythromycin, mosapride citrate, pantethine, panthenol, prostaglandin $\mathrm{F} 2 \alpha$, digestive enzyme prepara- 
tions, anticholinergic drugs, cathartic drugs, antidiarrheal drugs, antipsychotic drugs, lactobacillus preparation, anti-Parkinsonian drugs.

Limited drugs will be anti-emetic drugs such as metoclopramide, domperidone and DPP-4 inhibitor. Rescue drugs will be intestinal atony remedy, antidiarrheal drugs, cathartic drugs, and analgesic drugs.

\section{Test treatments}

This is a randomized, open, controlled study using patients treated with usual care as the control group. Patients who meet the inclusion criteria are randomized to the study groups. Eligible patients are randomized to the DKT therapy group (DKT administration for 14 days postoperatively or until the day of discharge if a patient leaves the hospital less than 14 days after the surgery) or the usual care group (no administration of DKT (ratio 1:1).

The design of present study is in accordance with the Standard Protocol Items: Recommendations for Interventional Trials (SPIRIT) and Consolidated Standards of Reporting Trials 2010 guidelines [22,23]. This is an investigator-initiated, randomized, open, controlled study of the effectiveness and safety of DKT compared with non-treatment. The study design is illustrated in Fig. 1.

\section{Arm A:}

Oral administration of DKT $5.0 \mathrm{~g}(2.5 \mathrm{~g} /$ pack $\times 2$ packs) per dose three times a day from postoperative day 1 to 14 .

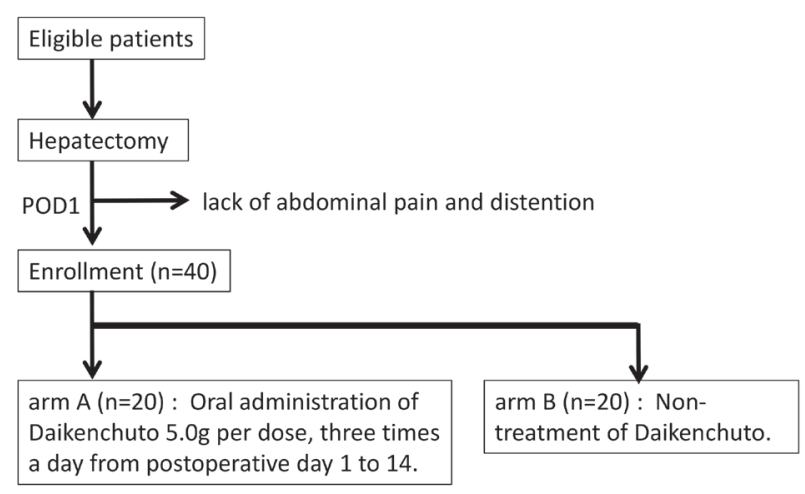

Fig. 1. Study Schema

Informed consent to be obtained by investigators from eligible patients before surgery. Patients are enrolled in the study if they can take DKT orally and have abdominal pain and/or bloating at postoperative day (POD) 1. Enrolled patients are randomly assigned to either the DKT group or non-treatment group.
Arm B: Non-treatment of DKT.

We monitor adherence by interviewing each patient and checking the number of trial drugs (DKT, Tsumura \& Co, Tokyo, Japan) that are administered . Patients and/or public were not involved in the design, recruitment, conduction and dissemination of the study.

\section{Endpoints}

The primary endpoint will be patient assessment of postoperative abdominal pain or bloating using NRS, and will be evaluated from day 1 to day 14 after the hepatectomy.

The following secondary endpoints will be also evaluated:

1) Postoperative hospital stay

2) Assessment of intestinal injury and nutritional status (DAO: Diamine oxidase; GLP-2: Glucagon-like peptide-2; RBP: Retinol-binding protein; TTR: Transthyretin; Tf; Transferrin; Alb: Albumin)

3) Liver function (ALT: Alanin-aminotransferase, AST: Aspartate transaminase, Al-P: Alkaline phosphatase, GGTP: Gamma-glutamyl transpeptidase, T-Bil: Total bilirubin, PT: Prothrombin)

4) Incidence and rate of postoperative complications (Clavien-Dindo Classification)

5) Incidence and rate of postoperative infection (wound, intraperitoneal, respiratory, urinary tract and systemic infection)

\section{Sample size}

Based on the results of previous clinical studies, DKT appeared to improve gastrointestinal dysmotility evaluated by time to first bowel movement [11]. The time to first bowel movement after liver resection was $88.2 \mathrm{~h}$ in the DKT group while it was $93.1 \mathrm{~h}$ in the placebo group. However, it has not been proven whether DKT is an effective treatment option for pain and bloating after liver resection in patients with liver cancer. Therefore, we estimated a sample size of 40 patients (20 patients in each group) as a pilot exploratory study to estimate safety and efficacy of DKT administration after hepatectomy for liver cancer.

\section{Study design}

After obtaining written informed consent and completing baseline data sets, the participants are enrolled and registered. All participants will be randomized to DKT or Non-treatment group at a 1:1 ratio 
(DKT vs. Non-treatment) centrally. The assignment factor was not set for this trial.

\section{Data collection}

Authorized clinical trial physicians and/or trial collaborators will prepare case report forms (CRFs). All CRF data must correspond to the original material if the data recorded directly in the CRF are not used as the material source. The investigators will obtain and collect data during the patient's hospital stay according to the time schedule in Fig. 2.

\section{Data management}

All study findings and documents will be recorded confidentially. Patients will be identified by their patient number in this trial and/or date of birth, not by name. Documents which could identify the patient must be confidentially preserved by the investigator. Therefore, the anonymity of participating patients is ensured.

Throughout the trial, protocol compliance, data source verification, assessment of drug accountability/ management, and assessment of laboratory procedures will be done by an in-house investigator. Therefore, the study shall be conducted according to pertinent regulation and regular protocol requirements.

\section{Statistical analysis}

The data importation in this study is performed us- ing SPSS Statistics Version 25 software (IBM, USA). Statistical tests will be two-sided, and $p$ values $<0.05$ will be regarded as significant for the primary endpoint. Statistical analyses are performed for the primary and the secondary endpoints.

Participants will be recruited at Nagasaki University Hospital, Nagasaki, Japan. Participants will hear an explanation of the study by responsible surgeons and the clinical research coordinators (CRC). Voluntarily signature for informed consent will be required before participation in the study.

\section{DISCUSSION}

We are evaluating the effectiveness and safety of DKT in preventing post-hepatectomy digestive symptoms using the NRS (numeric rating scale), and are examining the effects of DKT on small intestinal mucosal atrophy through an exploratory study using DAO and GLP-2 activities. The results of the study may provide clinical information on the effectiveness and safety of DKT and allow elucidation of the action mechanism of DKT in clinical practice. In addition, it is also expected to promote further exploration of improved treatments for damage to the intestinal mucosa and accompanying impairment of nutrient absorption capacity after gastroenterological surgery.

The study is being conducted according to the principles of the Declaration of Helsinki and Japanese

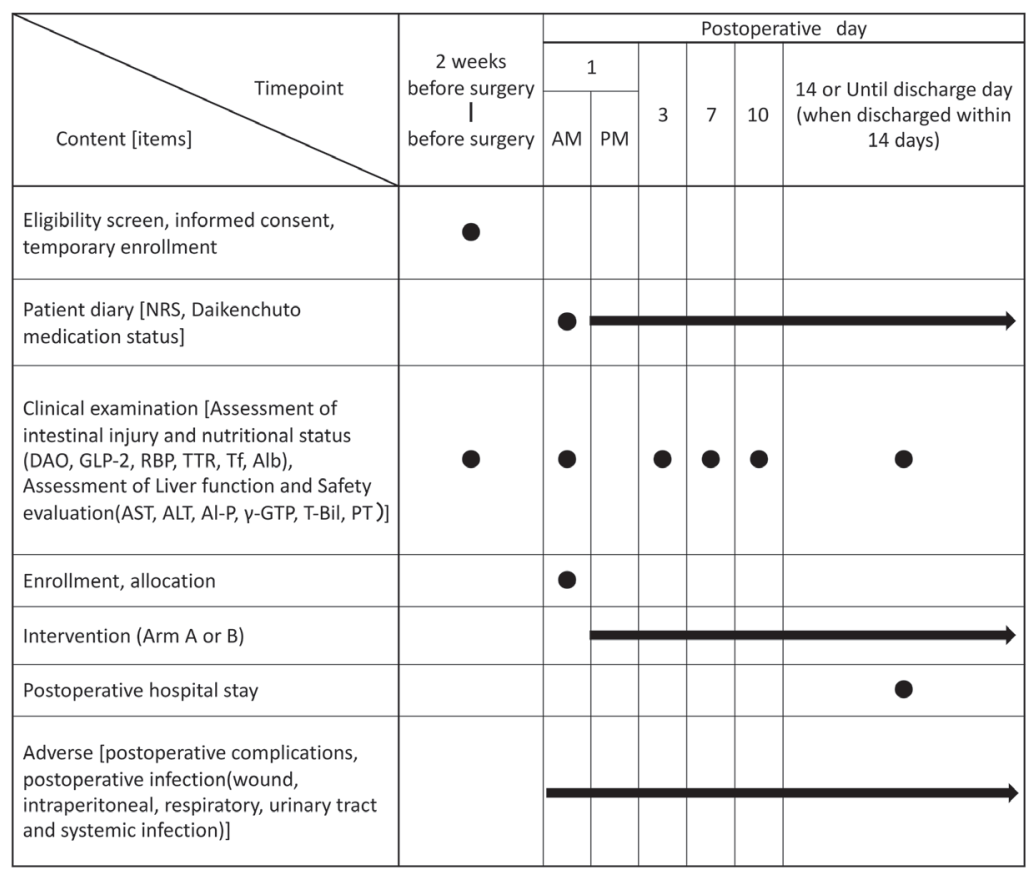

Fig. 2. Study Schedule

Schedule of enrolment, intervention and assessments. 
Ministerial Ordinance on Good Clinical Practice. The persons/institutions conducting the specified clinical study shall comply with the Helsinki Declaration (revised in October, 2013) and comply with the Clinical Trials Act (Act No. 16 of 2017) and the Regulation for Enforcement of the Clinical Act (Ordinance of the Ministry of Health, Labour and Welfare No. 17 of 2018).

\section{DECLARATION}

COMPETING INTERESTS: The authors declare that they have no conflict of interests.

FUNDING: This work was supported by a research grant from Tsumura \& Co., Tokyo, Japan.

AUTHORS' CONTRIBUTIONS: MH, AS and SE are responsible for conceiving of and designing the trial, planning g data analysis, drafting the manuscript, making the final decision to terminate the trial, and approving the final manuscript. $\mathrm{KN}$, SO, TA, YI and TK will participate in data collection and are in charge of recruitment and treatment of patients. SI and KK are responsible for planning data analysis and analyzing the data resulting fromal. All authors will have access to the interim results as well as the capacity to discuss, revise, and approve the final manuscript.

\section{ETHICS APPROVAL AND CONSENT TO}

PARTICIPATE: The Central Review Board and Local Ethics Committee (CRB7180001) approved the study protocol. All patients who fulfill the inclusion criteria will provide written informed consent.

CLINICAL TRIAL REGISTRATION: The trial started on 16 October, 2017 and data is currently being collected. There has not been any publication concerning the analysis of the data collected to date. The study is registered with the University Hospital Medical Information Network Clinical Trials Registry (www.umin.ac.jp/ctr/) under registration number UMIN000028950 and jRCTs071180028. Registered on 1 September, 2017.

\section{REFERENCES}

1. Kikuchi D, Shibata C, Imoto H, Naitoh T, Miura K et al. Intragastric Dai-Kenchu-To, a Japanese Herbal Medicine, Stimulates Colonic Motility via Transient Receptor Potential Cation Channel Subfamily V Member 1 in Dogs. Tohoku J Exp Med 2013; 230:197-204.

2. Satoh K, Hayakawa T, Kase Y, Ishige A, Sasaki H et al. Mechanisms for Contractile Effect of Dai-kenchu-to in Isolated Guinea Pig Ileum. Dig Dis Sci 2001; 46:250-256.

3. Satoh K, Kase Y, Hayakawa T, Murata P, Ishige A et al. Dai-kenchu-to Enhances Accelerated Small Intestinal Movement. Biol Pharm Bull 2001; 24:1122-1126.

4. Muraoka I, Takatsuki M, Soyama A, Yamaguchi I, Tanaka
$\mathrm{S}$ et al. Efficiency of herbal medicine Dai-kenchu-to on portal blood flow in rat models. Ann Med Surg 2015; 4:211-214.

5. Kono T, Kaneko A, Omiya Y, Ohbuchi K, Ohno N et al. Epithelial transient receptor potential ankyrin 1 (TRPA1) -dependent adrenomedullin upregulates blood flow in rat small intestine. Am J Physiol Gastrointest Liver Physiol 2013; 304:G428-G436.

6. Hayakawa T, Kase Y, Saito K, Hashimoto K, Ishige A et al. Pharmacological Studies of the Effect of Dai-kenchu-to on Spontaneous Contraction of Isolated Rabbit Jejunum. J Smooth Muscle Res 1999; 35:55-62.

7. Kono T. Kaneko A, Hira Y, Suzuki T, Chisato N et al. Anticolitis and -adhesion effects of daikenchuto via endogenous adrenomedullin enhancement in Crohn>s disease mouse model. J Crohns Colitis 2010; 4:161-170.

8. Yaegashi M, Otsuka K, Itabashi T, Kimura T, Kato K et al. Daikenchuto Stimulates Colonic Motility after Laparoscopic-Assisted Colectomy. epatogastroenterology 2014; 61:85-89.

9. Kono T, Shimada M, Nishi M, Morine Y, Yoshikawa K, Katsuno $\mathrm{H}$ et al. Daikenchuto accelerates the recovery from prolonged postoperative ileus after open abdominal surgery: a subgroup analysis of three randomized controlled trials. Surg Today 2019; 49:704-711.

10. Akamaru Y, Takahashi T, Nishida T, Omori T, Nishikawa K et al. Effects of Daikenchuto, a Japanese Herb, on intestinal motility after total gastrectomy: a prospective randomized trial. J Gastrointest Surg. 2015; 19:467-472.

11. Shimada M, Morine Y, Nagano H, Hatano E, Kaiho T et al. Effect of TU-100, a traditional Japanese medicine, administered after hepatic resection in patients with liver cancer: a multi-center, phase III trial (JFMC40-1001). Int J Clin Oncol 2015; 20:95-104.

12. Nishi M, Shimada M, Uchiyama H, Ikegami T, Arakawa Y et al. The beneficial effects of Kampo medicine Dai-kenchu-to after hepatic resection: a prospective randomized control study. epatogastroenterology 2012; 59:2290-2294.

13. Kaido T, Shinoda M, Inomata Y, Yagi T, Akamatsu N et al. Effect of herbal medicine daikenchuto on oral and enteral caloric intake after liver transplantation: A multicenter, randomized controlled trial. Nutrition 2018; 54:68-75.

14. Hanazaki K, Ichikawa K, Munekage M, Kitagawa H, Dabanaka K et al. Effect of Daikenchuto (TJ-100) on abdominal bloating in hepatectomized patients. World $\mathrm{J}$ Gastrointest Surg 2013; 5:115-122.

15. Yoshikawa K, Kurita N, Higashijima J, Miyatani T, Miyamoto $\mathrm{H}$ et al. Kampo Medicine "Dai-Kenchu-To" Prevents Bacterial Translocation in Rats. Dig Dis Sci 2008; 53:1824-1831.

16. Chikakiyo M, Shimada M, Nakao T, Higashijima J, Yoshikawa K et al. Kampo medicine "Dai-kenchu-to" prevents CPT-11-induced small-intestinal injury in rats. Surg Today 2012; 42:60-67.

17. Yada K, Ishibashi H, Mori H, Morine Y, Zhu C et al. The Kampo medicine aikenchuto(TU-100)" prevents bacterial translocation and hepatic fibrosis in a rat model of biliary atresia. Surgery 2016; 159:1600-1611 .

18. Kamiya S, Nagino M, Kanazawa H, Komatsu S, Mayumi T 
et al. The Value of Bile Replacement During External Biliary Drainage: An Analysis of Intestinal Permeability, Integrity and Microflora. Ann Surg 2004; 239:510-517.

19. Sugawara G, Nagino M, Nishio H, Ebata T, Takagi K et al. Perioperative Synbiotic Treatment to Prevent Postoperative Infectious Complications in Biliary Cancer Surgery: A Randomized Controlled Trial. Ann Surg 2006; 244:706714.

20. Dubé PE and Brubaker PL. Frontiers in glucagon-like peptide-2: multiple actions, multiple mediators. Am J Physiol Endocrinol Metab. 2007; 293:E460-E465.

21. Liver Cancer Study Group of Japan. The General Rules for the Clinical and Pathological Study of Primary Liver Cancer. 4th ed. Tokyo: Kanehara \& Co., Ltd.; 2000. (In Japanese)

22. Chan AW, Tetzlaff JM, Altman DG, Laupacis A, Gøtzsche PC et al. SPIRIT 2013 Statement: Defining Standard Protocol Items for Clinical Trials. Ann Intern Med 2013; 158:200-207.

23. Schulz KF, Altman DG, and Moher D for the CONSORT Group. CONSORT 2010 Statement; updated guidelines for reporting parallel group randomized trials. BMJ 2010; 340:c332. 